\title{
Parálisis periódica hipopotasémica tirotóxica. Caso clínico
}

\author{
VICTORIA FRANTCHEZ, JOSÉ VALIÑO, \\ ANALÍA CARRACELAS, CARLOS DUFRECHOU
}

\section{Thyrotoxic hypokalemic periodic paralysis. Report of one case}

Thyrotoxic hypokalemic periodic paralysis is characterized by attacks of generalized weakness associated to hypokalemia in patients with hyperthyroidism. We report a 25-year-old man with a history of spontaneously relapsing episodes of muscular weakness, who consulted for a rapidly evolving upper and lower limb paresis. Hypokalemia associated to a primary hyperthyroidism was detected. Treatment with antithyroid drugs and potassium supplementation reverted symptoms and the episodes of acute muscular weakness did not reappear.

(Rev Med Chile 2010; 138: 1427-1430).

Key words: Hypokalemia: Hypokalemic periodic paralysis; Thyrotoxicosis.

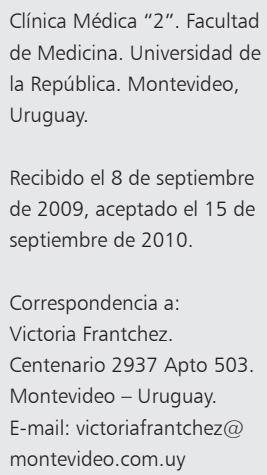

L a parálisis periódica hipopotasémica tirotóxica es una entidad de baja frecuencia, especialmente en Occidente, caracterizada por episodios de debilidad muscular aguda (DMA).

La DMA se presenta como una pérdida de fuerza generalizada, que se instala en pocas horas o días (menos de cuatro semanas), debida a una enfermedad neuromuscular. No incluye la pérdida de fuerza secundaria a enfermedades del sistema nervioso central.

La DMA puede ser causada por múltiples enfermedades. Dentro de este grupo se encuentran las parálisis hipopotasémicas agudas que son una emergencia terapéutica ${ }^{1,2}$.

Las parálisis hipopotasémicas se dividen en 2 grupos: las parálisis periódicas hipopotasémicas (PPH) secundarias a la entrada de $\mathrm{K}$ desde el espacio extracelular al intracelular y las parálisis hipopotasémicas no periódicas (PHNP) debidas a déficit del capital corporal total de $\mathrm{K}^{1,3}$.

Dentro de las PPH se encuentran: a) la parálisis periódica hipopotasémica familiar (PPHF), enfermedad autosómica dominante con mayor frecuencia en Occidente ${ }^{2,4}$, b) la parálisis periódica hipopotasémica esporádica (PPHE) y c) la parálisis periódica hipopotasémica tirotóxica (PPHT).

La PPHT es una complicación del hipertiroidismo con clara distribución geográfica, presentándose en 1,8-1,9\% de los pacientes hipertiroideos asiáticos ${ }^{5,6}$. Si bien el hipertiroidismo predomina en las mujeres, la PPHT es más frecuente en el sexo masculino ( $13 \%$ y $0,17 \%$ en hombres y mujeres respectivamente) $)^{5,6}$, entre los 20 y 40 años de edad ${ }^{5}$. Se han reportado casos en caucásicos, afro-americanos, indo-americanos e hispanos ${ }^{7}$ y en 0,1- 0,2 de los hipertiroideos en Estados Unidos de Norteamérica (EE.UU.) ${ }^{8,9}$. Dado los fenómenos de migración, en los últimos años hay un aumento en la incidencia de esta complicación en Occidente y América Latina ${ }^{7,10}$.

\section{Caso clínico}

Al Departamento de Emergencia del Hospital Pasteur (Montevideo, Uruguay), concurrió un paciente de sexo masculino de 25 años, de raza blanca, que consultó por debilidad muscular en miembros inferiores que se extendió en forma progresiva y simétrica a los miembros superiores 
y en menos de 6 horas le impidió la deambulación. No presentó alteraciones respiratorias, deglutorias, ni urinarias.

En los últimos tres meses había presentado episodios similares nocturnos, precedidos de calambres y mialgias, que remitían espontáneamente en menos de $24 \mathrm{~h}$, cuya frecuencia e intensidad aumentó en las semanas previas a la consulta. Relataba adelgazamiento, hiperorexia e irritabilidad en los últimos 6 meses, sin otros síntomas de hipertiroidismo. Su madre tenía diagnóstico de hipotiroidismo primario.

En la exploración física se destacaba: ansiedad, piel cálida y sudorosa, bocio difuso, taquicardia regular de $100 \mathrm{cpm}$, HTA sistólica $(180 / 80 \mathrm{mmHg})$ y cuadriparesia que no vencía la gravedad, a predominio de los miembros inferiores, simétrica, con hipotonía e hiporreflexia. La motilidad ocular y facial no presentaba alteraciones, la sensibilidad no estaba afectada y el resto de la exploración física era normal.

De la analítica sanguínea se destacaba una hipopotasemia de $2,5 \mathrm{mmol} / \mathrm{l}(3,5-5,0)$, hipomagnesemia de $0,56 \mathrm{mmol} / \mathrm{l}(0,65-1,03)$, hipofosfatemia de $2,3 \mathrm{mg} / \mathrm{dl}(2,5-4,5)$ y CPK total de $257 \mathrm{U} / \mathrm{l}(15-190)$.

El perfil tiroideo indicaba un hipertiroidismo primario, TSH $0,007 \mu \mathrm{IU} / \mathrm{ml},(0,23-4,20) \mathrm{T} 48,04$ ng/dl $(0,85-1,70)$, y T3 > 20 pgr/dl $(1,90-5,10)$. El centellograma tiroideo mostró un bocio difuso, con un peso aproximado de $43 \mathrm{~g}$, hipercaptante, compatible con enfermedad de Graves. Los anticuerpos antitiroideos no fueron solicitados.

Se formuló el diagnóstico de parálisis periódica hipopotasémica tirotóxica y se realizó tratamiento de urgencia con $\mathrm{KCl}$ i.v. revirtiendo rápidamente la debilidad muscular, con hiperpotasemia de rebote $\left(\mathrm{K}^{+}\right.$de $\left.5,8 \mathrm{mmol} / \mathrm{l}\right)$ a las $24 \mathrm{~h}$.

Además se indicó propanolol $40 \mathrm{mg}$ v.o. cada 8 h y metimazol $10 \mathrm{mg}$ v.o. cada $8 \mathrm{~h}$, no reiterando los síntomas ni las disionias.

\section{Discusión}

La asociación de una DMA periódica con síntomas de hipertiroidismo llevó al diagnóstico de PPHT. La parálisis recurrente, con afectación mayor de los músculos proximales, que se inicia en los miembros inferiores, extendiéndose a los superiores, respetando la motilidad facial y ocular, así como la inexistencia de compromiso de la sensibilidad y del sistema nervioso autónomo son características de esta enfermedad ${ }^{5}$.

La recuperación espontánea de la motilidad en menos de 24 horas es lo esperado en esta entidad, en la que la remisión se da con un patrón inverso, a las 3-72 h.

Las mialgias precediendo a la parálisis, al igual que la fatiga y los calambres son pródromos frecuentes ${ }^{5}$. La presentación nocturna de la mayoría de los episodios es lo habitual, pudiendo ser precipitados por la ingesta de carbohidratos y alcohol, ejercicio extenuante, infecciones o cirugía, entre otros ${ }^{5}$.

En la evaluación inicial un ionograma en sangre y orina confirmarán la hipopotasemia, descartando una pérdida urinaria de $\mathrm{K}$, con un metabolismo ácido-base normal.

La obtención de una muestra de orina en 24 h, en el Departamento de Emergencia es difícil por lo que se puede sustituir por la potasiuria en una muestra aislada de orina, concomitante con la hipopotasemia. Una potasiuria menor a $15 \mathrm{mEq} / \mathrm{l}$ en dicha muestra descartará una pérdida renal de $\mathrm{K}$ (en pacientes con diuresis normal) ${ }^{11}$. La intensidad de la hipopotasemia se correlaciona con el grado de parálisis. Se encuentra hipofosfatemia moderada en $2 / 3$ de $\operatorname{los}_{\text {casos }}{ }^{10}$ e hipomagnasemia secundaria al estado hiperadrenérgico ${ }^{5,10}$. El perfil tiroideo cerrará el diagnóstico (Figura 1).

La CPK se encontraba aumentada, (como se observa en $2 / 3$ de los casos), ya que en general existe rabdomiólisis y la fosfatasa alcalina se incrementa como consecuencia de la tirotoxicosis ${ }^{5,10}$.

El electrocardiograma puede mostrar como tríada diagnóstica de la PPHT: taquicardia sinusal (propia del estado hiperadrenérgico), prolongación del intervalo QT con aumento de la onda U (secundario a la hipopotasemia) y prolongación paradójica del intervalo PR (la taquicardia sinusal cursa con un PR corto) ${ }^{13}$.

Las hormonas tiroideas se encuentran en general moderadamente aumentadas, no correlacionándose con el grado de parálisis ${ }^{5}$. La PPHT es más frecuente en la enfermedad de Graves, si bien puede complicar otras enfermedades causantes de tirotoxicosis ${ }^{5,8}$.

El diagnóstico diferencial fue con la PPHF y la PPHE, de la que clínicamente pueden ser indistinguibles, dado que sólo 10\% de la PPHT se asocian a manifestaciones clínicas claras de tirotoxicosis ${ }^{14}$. La PPHF en general se presenta en menores de 20 


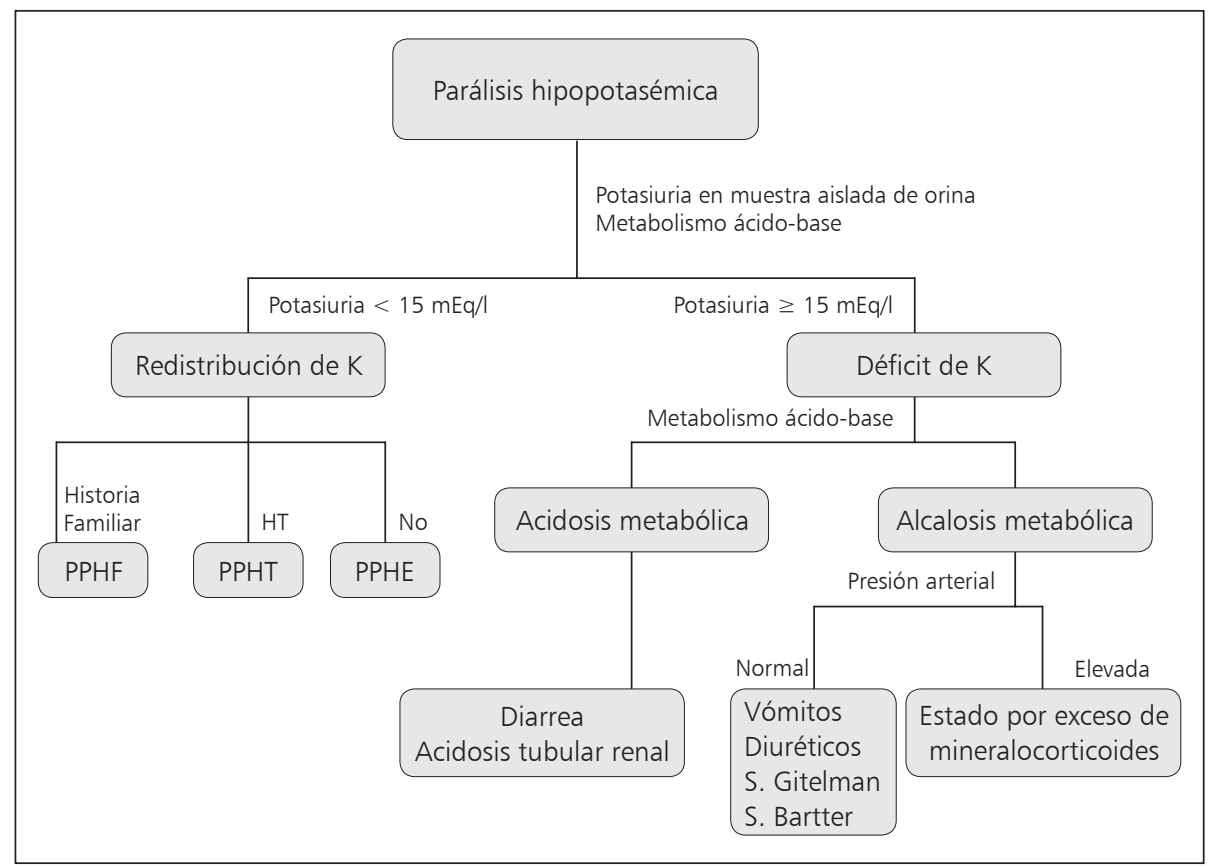

Figura 1. Algoritmo diagnóstico de Parálisis Hipopotasémica en el Departamento de Emergencias $^{12}$. Modificado de Lin $\mathrm{SH}$ et al. Laboratory test to determine the cause of hypokalemia and paralysis ${ }^{12}$. HT: hipertiroidismo. PPHF: Parálisis Periódica Hipopotasémica Familiar. PPHT: Parálisis Periódica Hipopotasémica Tirotóxica. PPHE: Parálisis Periódica Hipopotasémica Esporádica. años, no tiene predominancia de sexo y existe el antecedente familiar. La presencia de hipertensión, taquicardia e hipofosfatemia están a favor de la PPHT frente a la $\mathrm{PPHE}^{3}$.

La DMA en la PPHT se genera por la redistribución rápida y masiva del $\mathrm{K}$ del espacio extracelular al intracelular ${ }^{5}$ dependiente de un aumento de la actividad de la bomba Na/K ATPasa ${ }^{15,16}$. Esta bomba es estimulada por: a) las hormonas tiroideas de forma directa e indirecta a través de la estimulación del sistema adrenérgico ${ }^{5}$; b) la insulina (en estos pacientes se ha demostrado una respuesta exagerada en la secreción de insulina a las cargas de glucosa) ${ }^{17}$ y c) el ejercicio, que promueve la liberación de $\mathrm{K}$ al espacio extracelular ${ }^{8}$. Estos mecanismos explicarían que la dieta rica en carbohidratos y el ejercicio extenuante puedan desencadenar los ataques. La testosterona también promueve el ingreso del $\mathrm{K}$ al espacio intracelular (lo que explica la mayor prevalencia en hombres). Adicionalmente se postula que existe una mayor sensibilidad genética a los activadores de la bomba $\mathrm{Na} / \mathrm{K}$ ATPasa ${ }^{5}$.

El objetivo del tratamiento es en primer lugar prevenir arritmias potencialmente fatales secundarias a la hipopotasemia, prevenir el fallo respi- ratorio y revertir la parálisis muscular ${ }^{3,8}$. Se llevará el $\mathrm{K}$ a un rango de seguridad, no a la normalidad, para prevenir la hiperpotasemia de rebote que se presenta en $40 \%$ de los pacientes ${ }^{1,10,18}$. Se deberán evitar las soluciones glucosadas que estimulan la secreción de insulina agravando la hipopotasemia.

La reposición del potasio deberá tener en cuenta las siguientes pautas; si la potasemia es menor de $2,5 \mathrm{mEq} / \mathrm{l}$ se debe aportar $\mathrm{KCl}$ i.v. a un ritmo de $10 \mathrm{mEq} / \mathrm{h}$, si la potasemia es mayor del valor referido, se prefiere el aporte de K por vía oral, con $\mathrm{KCl} 2 \mathrm{~g}$ cada $2 \mathrm{~h}$ en las primeras $6 \mathrm{~h}$ o hasta que reviertan los síntomas, y luego cada $4 \mathrm{~h}$ hasta su normalización ${ }^{3,5}$. Es de destacar que dado que no existe una depleción del capital potásico corporal total, no se requieren importantes cantidades de $\mathrm{K}^{+}$para su corrección como en hipopotasemias de otra etiología.

Los agentes beta-bloqueantes no selectivos, como el propanolol, a una dosis de $3-4 \mathrm{mg} / \mathrm{kg}$, revierten rápidamente los síntomas y evitan la hiperpotasemia de rebote ${ }^{19}$. En la prevención de los ataques es importante evitar los factores precipitantes y se indicará propanolol $20-80 \mathrm{mg}$ cada 8 hs v.o. hasta el tratamiento definitivo del hipertiroidismo. 
En suma: Consideramos de interés la presentación de este caso de parálisis periódica hipopotasémica tirotóxica con el objetivo de difundir el conocimiento de un cuadro clínico cuya incidencia en los países no asiáticos está en aumento por los fenómenos migratorios, y resaltar algunos de sus aspectos clínicos y fisiopatológicos. Destacamos la importancia de tener presente esta enfermedad, difícil de diferenciar clínicamente de otras formas de parálisis periódica hipopotasémica y jerarquizamos la importancia de su diagnóstico precoz que permita iniciar rápidamente su tratamiento específico.

Agradecimientos: A Dr. Rafael Mila.

\section{Referencias}

1. Lin SH, Lin YF, Halpering ML. Hypokalemia and paralysis. QJM 2001; 94: 133-9.

2. Stedwell RE, Allen KM, Binder LS. Hypokalemic paralyses: A review of the etiologies, pathophysiology, presentation and therapy. Am J Emerg Med 1992; 10: 143-6.

3. Lin YF, Wu CC, Pei D, Chu SJ, Lin SH. Diagnostic Thyrotoxic Periodic Paralysis in the ED. Am J Emerg Med 2003; 21: 339-42.

4. Lapie P, Lory P, Fontaine B. Hypokalemic periodic paralysis: an autosomal dominant muscle disorder caused by mutations in a voltage-gated calcium channel. Neuromuscul Disord 1997; 7: 234-40.

5. Kung AW. Thyrotoxic periodic paralysis: A diagnostic challenge. J Clin Endocrinol Metab 2006; 91: 2490-5.

6. McFadzean AJS, Yeung R. Periodic paralysis complicating thyrotoxicosis in Chinese. Br Med J 1967; 1: 451-5.

7. Días DA, Silva MR, Chiamolera MI, Kasamatsu TS, Cerutti JM, Maciel RM. Paralisia Periódica Hipocalêmica Tirotóxica, Uma Urgencia Endócrina: Revisão do Quadro Clínico e Genético de 25 Pacientes. Arq Bras Endocrinol Metab 2004; 48: 196-215.

8. Ober KP. Thyrotoxic periodic paralysis in the United
States: report of 7 cases and review of the literature. Medicine 1992; 71: 109-20.

9. Pichon B, Lidove O, Delbot T, Aslangul E, Hausfater P, Papo T. Thyrotoxic periodic paralysis in Caucasian patients: A diagnostic challenge. Eur J Intern Med 2005; 16: 372-4.

10. Manoukain MA, Foote JA, Crapo LM. Clinical and metabolic features of thyrotoxic periodic paralysis in 24 episodes. Arch Intern Med 1999; 159: 601-6.

11. Groeneveld JH, Sijpkens YW, Lin SH, Davids MR, Halperin ML. An approach to the patient with severe hypokalemia: the potassium quiz. Q J Med 2005; 98: 305-16.

12. Lin SH, Lin YF, Chen DT, Chu P, Hsu CW, Halperin ML. Laboratory Tests to Determine the Cause of Hypokalemia and Paralysis. Arch Intern Med 2004; 164: 1561-6.

13. Goldberger ZD. An Electrocardiogram Triad in Thyrotoxic Hypokalemic Periodic Paralysis. Circulation 2007; 115: e179-e180.

14. Ko GTC, Chow CC, Yeung VTF, Chan HHL, Li JKY, Cockram CS. Thyrotoxic periodic paralysis in a Chinese population. Q J Med 1996; 89: 463-8.

15. Khan FA, Baron DN. Ion flux and Na-, K-ATPase activity of erythrocytes and leucocytes in thyroid disease. Clin Sci 1987; 72: 171-9.

16. Chan A, Shinde R, Chow CC, Cockram CS, Swaminathan R. In vivo and in vitro sodium pump activity in subjects with thyrotoxic periodic paralysis. Br Med J 1991; 303: 1096-9.

17. Lee KO, Taylor EA, Oh VM, Cheah JS, Aw SE. Hyperinsulinaemia in thyrotoxic hypokalemic periodic paralysis. Lancet 1991; 337: 1063-4.

18. Lu KC, Hsu YJ, Chiu JS, Hsu YD, Lin SH. Effects of potassium supplementation on the recovery of thyrotoxic periodic paralysis. Am J Emerg Med 2004; 22: 544-7.

19. Birkhahn RH, Gaeta TJ, Melniker L. Thyrotoxic periodic paralysis and intravenous propranolol in the emergency setting. J Emerg Med 2000; 18: 199-202.

20. López JM, Carrasco CM. ¿Pseudohipoparatiroidismo o déficit de vitamina D? Rev Med Chile 2004; 132: 1527 31. 